\title{
Phantom limb sensations and phantom limb pain in child and adolescent amputees
}

\author{
Krista L. Wilkins ${ }^{\mathrm{a}, *}$, Patrick J. McGrath ${ }^{\mathrm{b}}$, G. Allen Finley ${ }^{\mathrm{a}, \mathrm{c}}$, Joel Katz ${ }^{\mathrm{d}}$ \\ ${ }^{a}$ Pain Research Laboratory, Department of Psychology, Dalhousie University, Halifax, Nova Scotia B3H 4J1, Canada \\ ${ }^{\mathrm{b}}$ Department of Psychology, Psychiatry, Pediatrics and Occupational Therapy, Dalhousie University, Halifax, Nova Scotia B3H 4JI, Canada \\ ${ }^{\mathrm{c}}$ Department of Anaesthesia, IWK Grace Health Centre and Dalhousie University, Halifax, Nova Scotia B3J 3G9, Canada \\ ${ }^{\mathrm{d}}$ Department of Psychology, Toronto Hospital, Acute Pain Research Unit and Department of Anaesthesia, The Toronto Hospital and Mount Sinai Hospital, \\ Departments of Public Health Sciences and Anaesthesia, University of Toronto, Toronto, Ontario, Canada
}

\begin{abstract}
Objective: To provide a better understanding of the prevalence, correlates and quality of phantom sensations and phantom pain in child and adolescent amputees. Design: Retrospective survey study. Setting: Recruitment through the War Amputations of Canada. Participants: Sixty child and adolescent amputees aged $8-18$ years who were missing a limb due to a congenital limb deficiency $(n=27)$ or surgery/trauma $(n=33)$. Main outcome measure(s): Questionnaire to assess the occurrence and correlates of phantom sensations and phantom pain. Results: Forty-two percent of the total sample reported phantom sensations; $7.4 \%$ of the congenital group and $69.7 \%$ of the surgical group $\left(\chi^{2}=23.70\right.$ with $1 \mathrm{df}, P<0.01$.) Twenty-nine percent of the total sample reported phantom pain; $3.7 \%$ of the congenital group and $48.5 \%$ of the surgical group $\left(\chi^{2}=14.67\right.$, with $\left.1 \mathrm{df}, P<0.01\right)$. Eighty-eight percent of the amputees with phantom pain had stump pain, while $35.3 \%$ had phantom pain that was similar to pre-operative pain and $76.5 \%$ experienced pains other than phantom pain (e.g. headaches). Amputees identified exercise, objects approaching the stump, cold weather and 'feeling nervous' as the primary triggers of phantom sensations and/or phantom pain. Conclusion(s): Less than half of the sample experienced phantom sensations and phantom pain; however, the loss of a limb due to surgery is associated with an increase in the likelihood of experiencing these phenomena. (C) 1998 International Association for the Study of Pain. Published by Elsevier Science B.V.
\end{abstract}

Keywords: Phantom sensation; Phantom pain; Child; Adolescent; Amputee

\section{Introduction}

The term 'phantom limb' was coined in 1866 by Silas Weir Mitchell, who described phantoms as ghostly replicas of the lost limb, some of which are painful (i.e. phantom limb pain) and others which are not (i.e. phantom limb sensations) (Mitchell, 1866). While phantom sensations may be described, for example, as tingling or itchy, phantom pain consists primarily of burning, cramping and shooting pains (e.g. Sherman, 1994), or a pain similar to pre-operative pain (Jensen et al., 1983, 1985; Katz and Melzack, 1990). Phantom sensations and phantom pain typically begin within days of the amputation, and they tend to decrease in frequency and duration over time (Jensen et al., 1983, 1985).

\footnotetext{
* Corresponding author. Tel.: +1 902 4941938; fax: +1 9024946585 .
}

Despite the considerable amount of research on phantom sensations and phantom pain in adult amputees, there is little information on child and adolescent amputees. To date, the evidence includes several case studies and clinicbased studies in which some amputees with congenital limb deficiencies report a phantom limb (Weinstein and Sersen, 1964; Poeck, 1964; Saadah and Melzack, 1994), or phantom pain (Sohn, 1914). Phantoms are, however, more common in children with traumatic amputations (e.g. accident); $100 \%$ of these amputees report phantom sensations (Ritchie, 1980; Krane and Heller, 1995), while 83-100\% experience phantom pain (Krane and Heller, 1995; Ritchie, 1980, respectively). More recently, Melzack et al. (1997) reported that phantoms were experienced by $33 \%$ of individuals who were either born without a limb $(12 \%)$ or underwent surgical amputation prior to 6 years of age (21\%). 
Several attempts have been made to formulate a theoretical basis for phantom sensations and phantom pain. The proposed mechanisms include psychological factors, such as personality traits (Parkes, 1973), and neurophysiological mechanisms (e.g. peripheral, spinal cord, or central nervous system). Melzack (1989) proposed the concept of a neuromatrix to explain the sensation of a limb after amputation. It has also been suggested that the activity in the somatosensory cortical area of the brain may activate cortical areas close to areas that represent the amputated part (Merzenich et al., 1984). This 'remapping' of cortical areas (Merzenich et al., 1984) or perhaps, changes in sympathetic activity as triggered by emotions, events, or thoughts (Katz, 1992a,b) may account for changes in the sensations of the phantom limb (e.g. paraesthesia) upon stimulation of different body parts.

Past research provides little information about the sensory characteristics of phantom sensations and phantom pain, especially in child and adolescent amputees. We undertook a survey study to determine the prevalence and quality of phantom sensations and phantom pain in child and adolescent amputees, comparing amputees with congenital limb deficiencies and amputees following surgical amputation. We also examined physical (e.g. weather), psychosocial factors (e.g. intense feelings), and individual characteristics (e.g. presence of stump pain, pre-operative pain, or pains other than phantom pain) that may influence the experience of phantom sensations and phantom pain. With this information, we may be able to better determine the factors predicting who will experience only phantom sensations and who will develop phantom pain; this, in turn, may help to develop prevention and treatment programs.

\section{Subjects and methods}

\subsection{Participants}

The participants were 60 child and adolescent amputees ( 35 boys and 25 girls) ranging in age from 8 to 18 years (mean, 12.55; SD, 2.29). The participants were recruited through the War Amputations of Canada from the Child Amputee Program (CHAMP). Amputees with multiple amputations were excluded from the study because several questions in the questionnaire required the amputee to compare the phantom limb to the corresponding intact limb. There were 32 upper limb amputees (eight above elbow, 18 below elbow and six partial hand) and 28 lower limb amputees (three above knee, 15 below knee, one foot, four Syme's ${ }^{1}$ and five Van Nes Rotations ${ }^{2}$.

\footnotetext{
'The Syme's amputation is an amputation through the foot.

${ }^{2}$ With the Van Nes Rotation, 'the leg is restructured so that the foot of the affected leg is placed at the level of the of the knee of the sound leg and the foot is turned 180 degrees. The foot then functions as a knee joint and in effect, the heel becomes the knee and the ankle joint becomes the knee joint. The sole of the foot remains the weight-bearing surface within a specially designed prosthesis.' (War Amputations of Canada, 1995, p. 47).
}

Two hundred and forty-seven letters requesting participation were distributed; 60 to the Atlantic Provinces, 57 to Saskatchewan and Manitoba, and 130 to Alberta. Seventyfour (30.4\%) amputees responded and $60(24.3 \%)$ agreed to participate. Of the 14 parents who declined to allow their child to participate, five parents stated their child was born with a congenital limb deficiency and had no phantom sensations or phantom pain, two parents assumed that since their child never complained of phantom sensations or phantom pain he or she didn't have them, one parent feared that talking about phantom sensations and phantom pain would induce these phenomena in their child who had not previously experienced them, one parent was not interested in participating, one child was non-verbal, one family did not have a telephone, and three letters were returned to the investigators due to address changes.

The participants included child and adolescents who were missing a limb because of a congenital limb deficiency ( $n=$ 27 , accident $(n=16)$, cancer $(n=7)$, or other medical problems (e.g. club feet, meningitis) $(n=10)$. The groups were then combined into a congenital group $(n=27)$ and a surgical group $(n=33)$, with accident, cancer and medical problems making up the surgical group. The surgical group was created because, regardless of the degree of trauma, all amputees in this group had had a limb at some point in their life, whereas the congenital group had neither a limb, nor did they experience any trauma associated with the missing limb. One amputee in the surgical group was an amputee with congenital limb deficiency who had a tiny finger bud removed. The phantom sensations and phantom pain reported by this amputee were limited to this area and thus, he was included as a surgical amputee. The surgical and congenital amputees did not differ in their age at the time of the study. The average age of the congenital group was 12.37 (SD 2.63) and the average age of the surgical group was 12.70 (SD 2.40), $T(58)=-0.50, P=0.62$. The congenital and surgical amputees did not differ in gender. The congenital group consisted of 15 boys and 12 girls and the surgical group consisted of 20 boys and 13 girls, $\chi^{2}=$ 0.16 with $1 \mathrm{df}, P=0.70$. On average, the congenital group had been missing a limb for more years prior to the study (12.37 (SD 2.63)) than the surgical group (6.58 (SD 4.32)), $T(58)=6.10, P<0.05$.

\subsection{Procedure}

The participants and their parents received a letter outlining the nature and purpose of the study. Upon return of their consent form, a questionnaire assessing phantom sensations and phantom pain was mailed to those who agreed to participate. The questionnaire contained items on demographics, details surrounding the amputation, stump problems, and prosthesis use. Amputees who experienced phantom sensations and/or phantom pain completed questions concerning the location, quality, position, duration and intensity of these phenomena. There were also questions 
aimed at identifying the triggers of phantom sensations and phantom pain and determining how much each factor increased or decreased phantom sensations and/or phantom pain on a rating scale of 1 to $10(1=$ little increase or decrease and $10=$ strongest imaginable increase or decrease). Phone interviews were then conducted to resolve any uncertainties and to collect the data.

Approval for this study was obtained from the Dalhousie University Psychology Department Ethics Committee and the IWK Grace Health Centre Research Ethics Board.

\subsection{Statistical analyses}

Descriptive statistics, primarily percentages and averages, were used to describe the participants and their experiences of phantom sensations and phantom pain. A chi-square analysis was computed to compare the prevalence of phantom sensations and phantom pain for congenital amputees vs. surgical amputees, and amputees who lost a limb at a young age with those who lost a limb at an older age. A chi-square analysis was also used to examine the relationship between age and the interval between the time of amputation and interview date. The amount by which a physical or psychosocial factor was identified as increasing phantom sensations or phantom pain was given a positive numerical rating, while the amount by which a factor decreased phantom sensations or phantom pain was given a negative numerical rating and the factors that had no effect were given a zero rating. To derive a mean rating of effect, the amount by which a factor increased or decreased the phantom sensations or phantom pain was then averaged across participants. One-sample $t$-tests were computed for each mean to test its significance from zero.

\section{Results}

\subsection{Phantom sensations}

As shown in Fig. 1, the prevalence of phantom sensations was lower for congenital amputees $(2 / 27(7.4 \%)$ than for surgical amputees $(23 / 33(69.7 \%)), \chi^{2}=23.70$ with $1 \mathrm{df}$, $P<0.01$ (overall prevalence $=25 / 60(41.6 \%)$ ) $($ see $>$ Fig. 1). The surgical group was divided into amputees who lost a limb at or before the age of 6 years (young amputees) and those who lost a limb after the age of 6 years (older amputees). The proportion of young amputees reporting phantom sensations $(9 / 19(47.4 \%))$ was significantly lower than that of older amputees $(14 / 14(100.0 \%)), \chi^{2}=10.57$ with $1 \mathrm{df}$, $P<0.01$. Young amputees, however, also reported a longer mean interval between the time of amputation and interview date (mean, 7.33; SD, 2.12) than older amputees (mean, 2.64; SD, 2.47), $T=4.69$ with $21 \mathrm{df}, P<0.01$.

There were eight upper limb amputees and seventeen lower limb amputees with phantom limbs. The most common position of the phantom limb for upper limb amputees

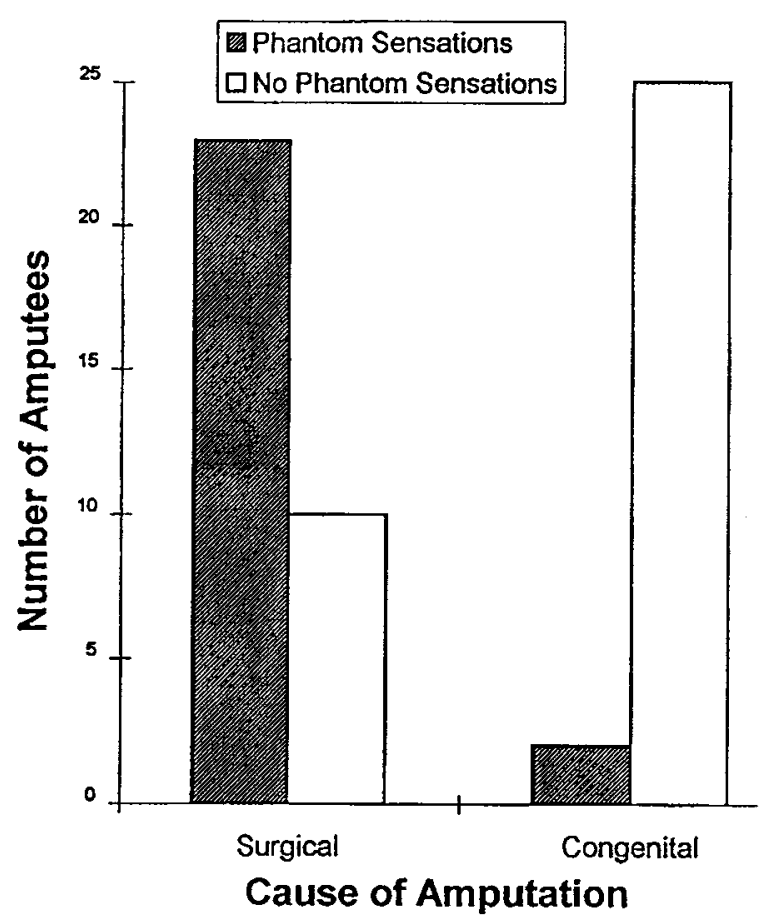

Fig. 1. Forty-two percent of the total sample reported phantom sensations; $7.4 \%$ of the congenital group and $69.7 \%$ of the surgical group $\left(\chi^{2}=23.70\right.$ with $1 \mathrm{df}, P<0.01$ ).

was fingers clenched in a fist $(4 / 8,50 \%)$, while the phantom limb of lower limb amputees was most commonly described as toes flexed $(8 / 17,47 \%)$. The phantom limb was shorter than the corresponding limb for nine participants $(36.0 \%)$, the same length as the corresponding limb for 15 participants $(60.0 \%)$ and its length varied for one participant $(4.0 \%)$.

The most common descriptors of the phantom sensations were tingling (60\%), numb (52\%), itchy $(40 \%)$, 'feels as if asleep' $(40 \%)$ and tickling (32\%). Phantom sensations occurred constantly in six $(24.0 \%)$ of the participants, once a day or more often in five $(20.8 \%)$, once a week or more often in six $(24.0 \%)$ and once a month or more often in eight $(32.0 \%)$. Phantom sensations were usually short in duration, lasting only seconds in six $(24.0 \%)$ of the participants, minutes in $13(52.0 \%)$, hours in three $(12.0 \%)$ and days in three $(12.0 \%)$. On average, the phantom sensations were rated as 5.32 (SD 2.36) on a rating scale of $0-10$, with $0=$ no sensation and $10=$ strongest imaginable sensation. Although phantom sensations were strong, all amputees reported that they did not interfere or caused little interference with daily activities (e.g. school, play). An object approaching the stump was rated as increasing phantom sensations significantly, $T(24)=2.27, P<0.05$. Similarly, 'feeling nervous' increased phantom sensations significantly, $T(24)=2.15, P<0.05$. There were, however, many triggers that were idiosyncratic (i.e. increased phantom sensations for some amputees and decreased phantom sensations for other amputees).

While only two amputees with congenital limb deficien- 
cies experienced phantom sensations, they described their phantom sensations as strong (mean, 7.00; SD, 4.24) and lasting minutes, occurring frequently as an itchy, numb, or heavy sensation. The most common triggers of their phantom sensations included not wearing a prosthesis, feeling happy, loud noises, and feeling cheerful.

All five of the amputees with a Van Nes Rotation reported having short, yet frequent episodes of phantom sensations with a mean strength of 3.20 (SD 2.55). The phantom sensations were referred to the missing knee and described as tingling, itchy, prickling, and 'feels as if asleep'. Not wearing a prosthesis, doing nothing, illness or colds, and feeling frightened increased their phantom sensations.

\subsection{Phantom pain}

As shown in Fig. 2, the prevalence of phantom pain was lower for the congenital group $(1 / 27(3.7 \%))$ than for surgical amputees $(16 / 33(48.5 \%)), \chi^{2}(1, n=60)=14.67, P<$ 0.01 (overall prevalence $=17 / 60(28 \%)$ ) (see Fig. 2$)$. The surgical group was divided into amputees who lost a limb at or before the age of 6 years (young amputees) and those who lost a limb after the age of 6 years (older amputees). The prevalence of phantom pain was lower for young amputees $(4 / 19(20.05 \%))$ than older amputees $(12 / 14(85.7 \%)), \chi^{2}=$ 13.49 with $1 \mathrm{df}, P<0.01$. As with phantom sensations, young amputees reported a longer mean interval between time of amputation and interview date (mean, 6.75; SD, 2.75) than older amputees (mean, 2.67; SD 2.46), $T=2.80$ with $14 \mathrm{df}, P<0.05$.

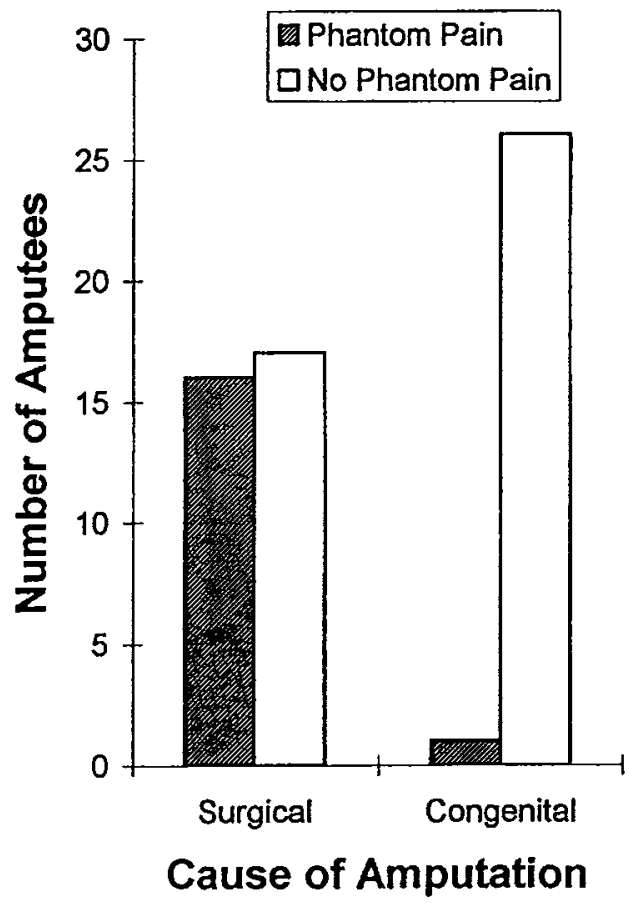

Fig. 2. Twenty-nine percent of the total sample reported phantom pain; $3.7 \%$ of the congenital group and $48.5 \%$ of the surgical group $\left(\chi^{2}=14.67\right.$ with $1 \mathrm{df}, P<0.01$ )
The most common descriptors of phantom pain included tingling $(53 \%)$, uncomfortable (47\%), pins and needles $(47 \%)$, throbbing (42\%), and sharp (42\%). Phantom pain was reported to have begun immediately following the amputation for six participants $(35.3 \%)$, within days for three $(17.6 \%)$, within weeks for one $(5.8 \%)$, within months for four $(23.5 \%)$, and within years for one $(5.8 \%)$. Phantom pain occurred constantly in three $(17.6 \%)$ of the participants, once a day or more often in three $(17.6 \%)$, once a week or more often in three $(17.6 \%)$, and once a month or more often in eight (47.2). Phantom pain was usually of short duration, lasting only seconds in six (35.3\%), minutes in seven (41.2\%), hours in two $(11.8 \%)$, and days in two $(11.8 \%)$ amputees. On average, the phantom pain was rated $5.29(\mathrm{SD}, 2.44)$ on a rating scale of $0-10$, with $0=$ no pain and $10=$ strongest imaginable pain. Even when phantom pain was strong, amputees reported that it either did not interfere or caused little interference with daily activities (e.g. school, play). Exercise increased phantom pain significantly, $T(16)=2.43, P<0.05$ as did an object approaching the stump, $T(16)=2.31, P<0.05$. Cold weather marginally increased phantom pain, $T(16)=2.08$, $P=0.054$. As with phantom sensations, there were many triggers that were idiosyncratic.

The prevalence of stump pain was $19 / 27(33.3 \%$ ) in the congenital group and $23 / 33(69.7 \%)$ in the surgical group. Stump pain occurred in $15 / 17(88.2 \%)$ of the amputees with phantom pain, while only $17 / 43(39.5 \%)$ of those without phantom pain had stump pain, thus suggesting that phantom pain and stump pain are indeed related $\left(\chi^{2}=11.61\right.$ with 1 df, $P<0.01)$. Pre-operative pain occurred in $6 / 17(35.3 \%)$ of amputees with phantom pain. Phantom pain was reported to be similar to pre-operative pain with respect to location, strength and quality for three $(50 \%)$ participants, to location only for two $(33.3 \%)$ participants and to quality only for one $(16.7 \%)$ participants and quality for four $(66.7 \%)$ participants. Pains other than phantom pain were experienced in $13 / 17(76.5 \%)$ of the amputees with phantom pain. The most common pains included headaches $(35 \%)$, pain in bones or joints $(35 \%)$, sore throat $(28 \%)$, stomach aches $(18 \%)$, and backaches $(12 \%)$. Thirteen $(76 \%)$ of the amputees reported that they ignore the phantom pain or attempt to decrease it by massaging the stump (29\%), taking medication (e.g. acetaminophen) $(12 \%)$, relaxing (12\%), or placing a heating pad on the stump (6\%).

While only one amputee with a congenital limb deficiency experienced phantom pain, this amputee reported having strong phantom pain episodes (mean 7.00). The phantom pain lasted for minutes, yet occurred relatively infrequent (i.e. once a month) and they were described as throbbing, uncomfortable, sore, itchy and spasm-like. Touching the stump, prosthesis usage, and dreams triggered the phantom pain, which was relieved only by ignoring it. This amputee also reported having stump pain and pains other than phantom pain.

Three of the five amputees with a Van Nes Rotation 
reported having short (i.e. minutes), yet frequent episodes of phantom pain with a mean strength of 3.33 (SD 1.15). As with phantom sensations, the phantom pain was referred to the missing knee. It was most commonly described as pins and needles, tingling, aching, and uncomfortable. Exercise, objects approaching the stump, prosthesis usage, touching the stump, and doing nothing increased their phantom pain. Two of these amputees reported having stump pain, while all three had pains other than phantom pain.

\section{Discussion}

Phantom sensations and phantom pain occur in child and adolescent amputees. While less than half of the sample experienced phantom sensations and/or phantom pain, the loss of a limb due to surgery was associated with a dramatic increase in the likelihood of an amputee experiencing these phenomena. In fact, $69.7 \%$ of the surgical group had phantom sensations and $48.5 \%$ had phantom pain, whereas only $7.4 \%$ and $3.7 \%$ of the congenial amputees had phantom sensations and phantom pain respectively. Similarly, Melzack et al. (1997) found that a greater number of amputees with surgical amputations had phantoms than amputees with congenital limb deficiencies.

These findings are, however, difficult to interpret given the greater time lapse between birth and the interview date for amputees with congenital limb deficiencies than the surgery and interview dates for the amputees with surgical amputations. One possibility to explain this difference is that a phantom is proportional to the intensity of input involved in the amputation; the more intense and traumatic the experience surrounding one's amputation, the more likely one is to experience a phantom. Another possible explanation is that phantoms of amputees with congenital limb deficiencies have had more time to fade or because of lack of use, central reorganization of processes may have taken over the brain regions subserved by the congenitally absent limbs.

The prevalence rates are lower than those found in the past. Krane and Heller (1995) found that phantom sensations occurred in $100 \%$ of their sample, whereas the prevalence of phantom pain was $83 \%$. However, Krane and Heller (1995) had only three amputees with congenital limb deficiencies, 12 amputees who lost a limb to trauma/infection, and 10 who lost a limb to cancer, while the our study had 27 amputees with congenital limb deficiencies, 16 amputees who lost a limb from an accident, seven amputees who lost a limb from cancer, and 10 amputees who lost a limb due to medical problems. Furthermore, we had a community sample, not a sample attending a clinic, and this may account for the lower prevalence rates.

This study contributes to the growing evidence that phantom limbs occur in amputees who are born missing a limb and those who lose a limb at an early age. Not only does this study support the finding that a small percentage of ampu- tees with congenital limb deficiencies experience phantom sensations, but it also confirms that some experience phantom pain. Young amputees reported a longer time interval between the time of amputation and interview date, and fewer episodes of phantom sensations and phantom pain than amputees older than six years of age. This age effect confirms Simmel's finding that the occurrence of phantoms increases with age at amputation and age at interview (Simmel, 1962).

Phantom pain resembled pre-operative pain with respect to location, quality and strength for $35.3 \%$ of the amputees. Krane and Heller (1995) found, however, that $75 \%$ of child amputees with phantom pain had pre-operative pain. The adult literature suggests that between 10-42\% (Nikolasjen et al., 1997; Katz and Melzack, 1990, respectively) of adults reported having pre-operative pain in the phantom limb.

Eighty-eight percent of the amputees who had phantom pain also had stump pain. This suggests that the relationship between phantom pain and stump pain is not limited to adult amputees. As well, amputees with phantom pain reported having pains other than phantom pain, but they were not frequent. Since we did not record the occurrence of other pains in amputees who did not have phantom pain, the relationship between phantom pain and other pain remains uncertain.

As a group, amputees were able to describe their sensations and to specify factors that commonly affect the occurrence of phantom sensations and phantom pain. Why do some factors increase phantom sensations in one amputee and decrease it in another? According to Katz (1992a,b), phantom sensations are related to a sympathetic-efferent somatic-afferent cycle. An increase in phantom sensations follows an increase in sympathetic activity, while a decrease in phantom sensations follows a decrease in sympathetic activity. These increases and decreases in sympathetic activity may be triggered centrally by a variety of intense events, emotions or thoughts. Most amputees reported efforts to decrease phantom pain by simply ignoring it.

The low response rate to the survey $(31.6 \%)$ is a problem because the results may not be representative of the child and adolescent population of amputees. The low response rate might be related to the parents' uncertainty of whether their child would be of any help to the study. It is not known however what specific population responded, but if we assume that approximately $60 \%$ of the amputees in CHAMP are amputees with congenital limb deficiencies (War Amputations of Canada estimate), we would expect there to be 148 amputees with congenital limb deficiencies and 99 surgical amputees in the targeted population. A conservative estimate of the prevalence of phantom sensations would then be $25 / 147(10.1 \%) ; 2 / 148(1.4 \%)$ for amputees with congenital limb deficiencies and $23 / 99(23.2 \%)$ for surgical amputees. The prevalence of phantom pain could be as low as $17 / 247(6.9 \%) ; 1 / 148(0.7 \%)$ for amputees with congenital limb deficiencies and 16/99 (16.2\%) for surgical amputees. 
This survey study was retrospective, and thus relied upon the amputees' memory. Future research could overcome this problem by the use of a prospective diary. Since phantom sensations and phantom pain do occur in child and adolescent amputees, this population warrants further investigation, especially in determining how to prevent and treat these phenomena.

\section{Acknowledgements}

This research was funded by an IWK Grace Health Centre Research grant to the first author. P.McG. is supported by an unrestricted grant from Bristol Myers Squibb and a Medical Research Council of Canada (MRC) Grant MT-13134. Joel Katz is supported by a MRC scholarship and MRC Grant MT-12052. We are indebted to those who kindly participated in the study and to the War Amputations of Canada for their help in recruiting participants.

\section{References}

Jensen, T.S., Krebs, B., Nielsen, J. and Rasmussen, P., Phantom limb, phantom pain, stump pain in amputees during the first six months following limb amputation, Pain, 17 (1983) 243-256.

Jensen, T.S., Krebs, B., Nielsen, J. and Rasmussen, P., Immediate and long-term phantom limb pain in amputees: incidence, clinical characteristics and relationship to pre-amputation limb pain, Pain, 21 (1985) 267-278.

Katz, J., Psychophysical contributions to phantom limbs, Can. J. Psychiatry, 37 (1992) 282-298.

Katz, J., Psychophysical correlates of phantom limb experience, J. Neurol. Neurosurg. Psychiatry, 55 (1992) 811-821.
Katz, J. and Melzack, R., Pain 'memories' in phantom limbs: review and clinical observations, Pain, 43 (1990) 319-336.

Krane, E.J. and Heller, L.B., The prevalence of phantom sensations and phantom pain in pediatric amputees, J. Pain Symptom Managem., 10 (1995) 21-29.

Mitchell, S.W., The case of George Dedlow, The Atlantic Monthly, 18 (1866) 1-11.

Melzack, R., Phantom limbs, the self and the brain (the D.O. Hebb memorial lecture), Can. J. Psychol., 30 (1989) 1-16.

Melzack, R., Isreal, R., Lacroix, R. and Schultz, G., Phantom limbs in people with congenital limb deficiency or amputation in early childhood, Brain, 120 (1997) 1603-1620.

Merzenich, M.M., Nelson, R.J., Stryker, M.P., Cynader, M.S., Schoppmann, A. and Zook, J.M., Somatosensory cortical map changes following digit amputation in adult monkeys, J. Comp. Neurol., 224 (1984) 591-605.

Nikolasjen, L., Ilkjaer, S., Kroner, K., Christensen, J.H. and Jensen, T.S., The influence of preamputation pain on postamputation stump and phantom pain, Pain, 72 (1997) 393-405.

Parkes, C.M., Factors determining the persistence of phantom pain in the amputee, J. Psychosom. Res., 17 (1973) 97-108.

Poeck, K., Phantoms following amputation in early childhood and in congenital absence of limbs, Cortex, 1 (1964) 276-290.

Ritchie, J.A., Nursing the child undergoing limb amputation, Am. J. Matern. Child Nurs., 5 (1980) 114-120.

Saadah, E.S.M. and Melzack, R., Phantom limb experiences in congenital limb-deficient adults, Cortex, 30 (1994) 479-485.

Sherman, R., Phantom limb pain: mechanism-based management, Pain Managem., 11 (1994) 85-106.

Simmel, M.L., Phantom experiences following amputation in childhood, J. Neurol. Neurosurg. Psychiatry, 25 (1962) 69-78.

Sohn, D.L., The psychic complex in congenital deformity, N.Y. Med. J., 100 (1914) 959-961.

War Amputations of Canada, Amputation, Ottawa, Ontario, 1995, 74 pp.

Weinstein, S., Sersen, E.A. and Vetter, R.J., Phantoms and somatic sensations in cases of congenital aplasia, Cortex, 1 (1964) 276-290. 